\title{
Détermination de l'effort de contact pantographe-caténaire par méthode inverse
}

\author{
Julien Berger ${ }^{1, a}$, Christian Clerc ${ }^{1}$, Stéphane Teppe $^{1}$ et Adrien Bobillot ${ }^{2}$ \\ 1 VIBRATEC, 28 chemin du Petit Bois, BP 36, 69131 Écully Cedex, France \\ 2 Direction de l'Ingénierie SNCF, Département Traction Électrique, 6 avenue François Mitterrand, \\ 93574 La Plaine Saint-Denis Cedex, France
}

Reçu le 2 October 2008, accepté le 23 juin 2009

\begin{abstract}
Résumé - La connaissance de l'effort de contact entre le pantographe et la caténaire est un élément essentiel de l'évaluation de la qualité du captage du courant. La SNCF étudie cet effort pour qualifier le matériel et les infrastructures nouvelles, ou en vue de détecter les défauts dans la caténaire. La méthode de mesure utilisée jusqu'à présent, basée sur une hypothèse d'archet rigide, a une bande passante limitée bien en deçà de la première fréquence propre de l'archet. Pour franchir cette limitation, notamment pour les trains à grande vitesse, et pour la détection de défaut par analyse de la signature vibratoire, une nouvelle méthodologie, basée sur la prise en compte des modes propres de l'archet, est proposée. Vibratec développe depuis plusieurs années des méthodes inverses [6,7] pour remonter aux efforts injectés à partir des vibrations mesurées. L'originalité du contact pantographe caténaire réside dans le fait que la position du point de contact est une inconnue du problème. La méthode inverse utilisée est basée sur l'analyse modale des premiers modes de l'archet. L'écriture de l'équilibre dynamique de l'archet permet d'aboutir à un système d'équations dont la résolution donne le module de l'effort et son point d'application. Les résultats obtenus sur un dispositif expérimental de validation, à partir d'une base modale limitée aux 3 premiers modes de l'archet, montrent un gain important sur la bande utile de la mesure, qui passe de $45 \mathrm{~Hz}$ à $120 \mathrm{~Hz}$. Par la suite, la bande passante pourra encore être étendue en considérant davantage de modes de déformation de l'archet. Un utilitaire a été développé sous Matlab pour la mise en œuvre de la méthode à partir de données expérimentales.
\end{abstract}

Mots clés : Transport ferroviaire / pantographe / ligne caténaire / archet / effort de contact / méthode inverse / usure / captage courant / signature vibratoire / analyse modale

\begin{abstract}
Contact force measuring method on a pantograph by inverse method. The catenary pantograph contact force is an essential element for railway electric power supply. SNCF needs to know this effort for new trains and networks design but also for catenary defaults detection. The current used measurement technique is based on a stiff bow assumption, which restricts the frequency band validity under the bow first natural frequency. This limitation must be overtaken for high speed and defaults detection; therefore, an inverse method, based on a bow modal analysis, is applied. Vibratec has a large background in inverse methods development and application $[6,7]$ but the catenary pantograph specificity is that the contact location on the bow is unknown. The bow dynamic equilibrium equations allow to compute the level and the location of the contact force. The experimental results, obtained from a dedicated test bench, show a large increase of the useful frequency band, which moves from $45 \mathrm{~Hz}$ to $120 \mathrm{~Hz}$. In the future, this band could be enlarge by using additional bow deformation modes. A Matlab application is achieved for post-processing the measured data and calculating the contact force.
\end{abstract}

Key words: Railway transportation / pantograph / catenary line / bow / contact force / inverse method / wear / electric power supply / dynamics / vibratory signature / modal analysis

a Auteur pour correspondance : julien.berger@vibratec.fr 


\section{Introduction}

La connaissance de l'effort de contact entre le pantographe et la caténaire est un élément essentiel de l'évaluation de la qualité du captage du courant. La SNCF étudie ainsi cet effort pour qualifier le matériel et les infrastructures nouvelles, ou en vue de détecter les défauts dans la caténaire.

La méthode utilisée jusqu'à présent, pour déterminer l'intensité de cet effort en ligne, est basée sur la mesure du comportement dynamique de l'archet supposé rigide, ce qui limite la bande passante en deçà de la première fréquence propre de l'archet.

Il était nécessaire de franchir cette limitation pour les trains à grande vitesse et pour la détection de défaut par analyse de la signature vibratoire.

Pour accroître la bande passante de mesure, une nouvelle méthodologie, basée sur la prise en compte des modes propres de l'archet, est proposée pour la détermination de l'effort de contact.

Vibratec développe depuis plusieurs années des techniques pour résoudre les problèmes inverses et remonter aux efforts injectés aux structures à partir des réponses vibratoires mesurées [6,7]. En général, les points d'entrée des efforts sont connus et on cherche à en déterminer les composantes ; la particularité du problème du contact caténaire-pantographe réside dans le fait que le point de contact se déplace continuellement sur la largeur de l'archet. Il est donc nécessaire de bâtir un système d'équation qui relie des déplacements mesurés sur l'archet à l'effort et à la position de contact.

\section{2 État de l'art}

Les méthodes actuelles pour mesurer l'effort de contact «archet-caténaire » supposent un archet indéformable. L'effort ainsi mesuré est correct jusqu'à environ 20-30 Hz. Au-delà de cette fréquence, la mesure est entachée d'erreurs principalement dues à la déformation de l'archet.

Des travaux réalisés par le « Railway Technical Research Institute » japonais [1] ont montré que le fait de considérer les premiers modes de déformation de l'archet permettaient d'augmenter la validité de la mesure jusqu'à environ $100 \mathrm{~Hz}$. L'appréciation de l'effort de contact est ensuite faussée à cause de la non-prise en compte des efforts aérodynamiques. La méthode japonaise s'appuie sur le principe de superposition modale et la mesure de diverses grandeurs :

- accélérations de l'archet par plusieurs accéléromètres;

- déformation de l'archet par deux jauges de déformation.

En France, l'instrumentation pour déterminer l'effort de contact par la méthode « archet indéformable » est composée de :

- deux accéléromètres localisés sur les boîtes à ressort;

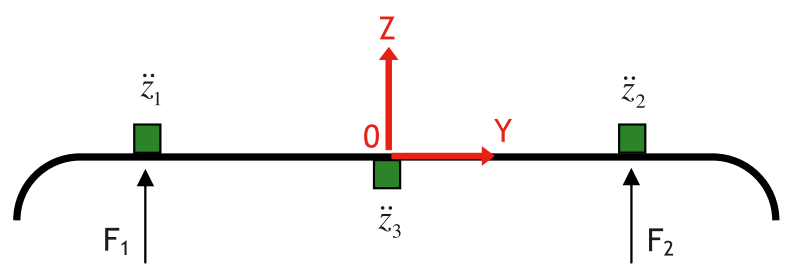

Fig. 1. Instrumentation envisagée.

- deux mesures d'efforts transitant dans les boîtes à ressort $^{1}$.

La nouvelle méthode, que proposent ici VIBRATEC et la SNCF, permet de conserver cette instrumentation.

\section{Nouvelle méthodologie}

\subsection{Systéme considéré et instrumentation envisagée}

Le système considéré est un archet soumis à trois efforts extérieurs :

- l'effort de contact;

- les efforts de réaction dans les boîtes à ressort.

L'instrumentation utilisée est la suivante (Fig. 1) :

- deux accéléromètres placés au niveau des boîtes à ressort;

- accéléromètres placés au centre de l'archet (un sur chaque bande de frottement);

- mesure d'effort dans les boîtes à ressort.

\subsection{Hypothèses}

Quel que soit le type d'archet (bi-bandes ou monobande), l'effort de contact recherché est un effort global qui s'applique sur les bandes de frottement. À ce jour, aucune distinction sur les efforts de contact n'est apportée pour les archets bi-bandes (i.e. effort sur bande $\mathrm{n}^{\circ} 1$, effort sur bande $n^{\circ} 2$ ). Le système à résoudre est donc un système plan.

Les archets ont été caractérisés par une analyse modale expérimentale en configuration « libre-libre ». Cette configuration est équivalente à l'archet monté sur ces boîtes à ressort car la fréquence du mode de suspension verticale de l'archet sur les boîtes à ressort est très inférieure à la fréquence du premier mode de déformation élastique. Le premier mode élastique de l'archet en configuration « libre-libre » est identique à la configuration de l'archet monté sur le pantographe : même amplitude et déformée modale.

\footnotetext{
1 Une boîte à ressort est composée d'un corps solidaire au bras du pantographe, d'un axe coulissant fixé sur l'archet et d'un ressort entre ces deux composants. Pour la mise en œuvre de la méthode, cette boîte est instrumentée de capteur d'ef-
} forts.

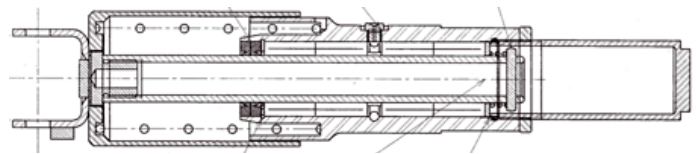




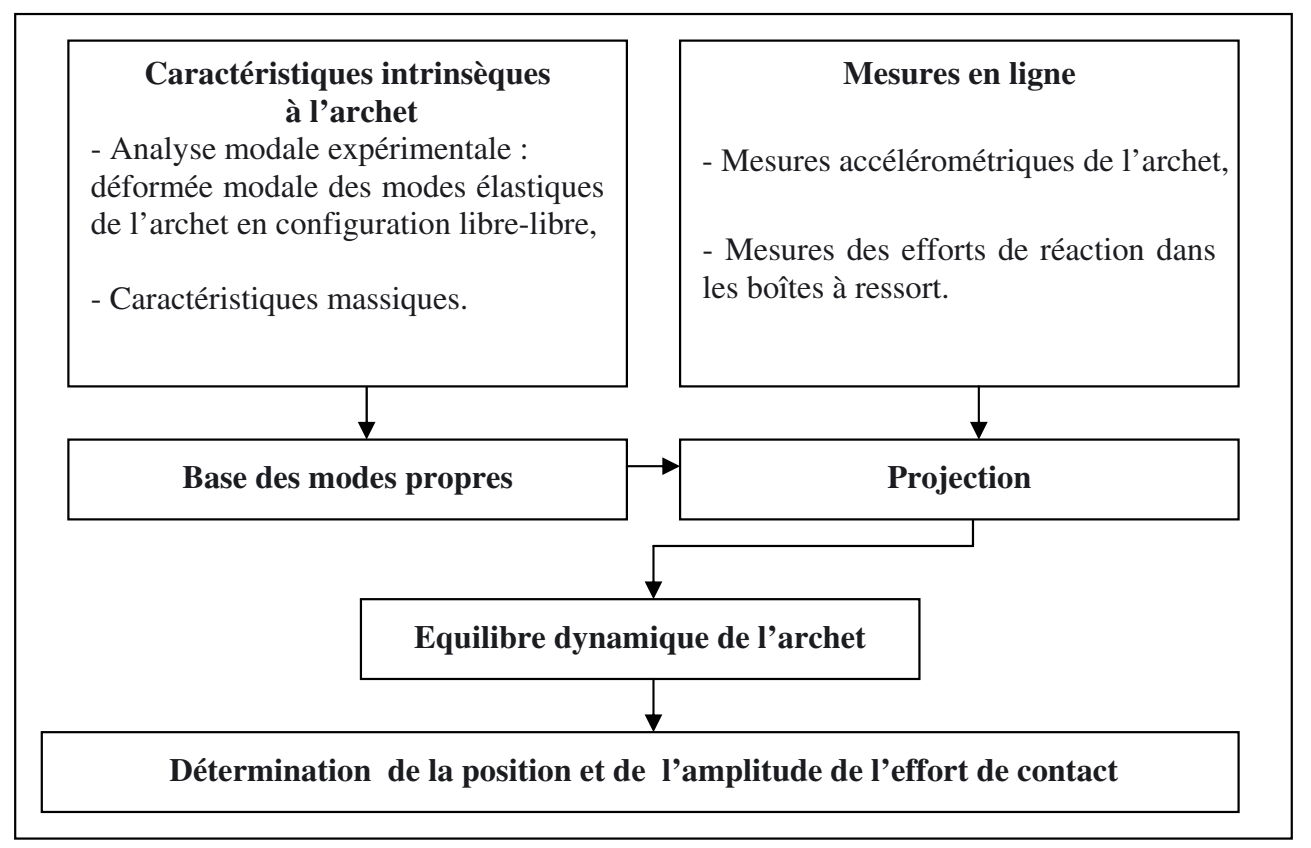

Fig. 2. Détermination de l'effort de contact.

\subsection{Méthodologie}

La méthode proposée est basée sur le principe de superposition modale inverse. Cette méthodologie suppose que les premiers modes élastiques de l'archet sont initialement connus.

L'écriture de l'équilibre dynamique de l'archet, soumis aux trois efforts extérieurs et projeté sur la base des modes propres, permet d'aboutir à un système d'équations dont l'amplitude et le point d'application de l'effort peuvent être extraits.

Le synoptique suivant, figure 2, illustre le cheminement pour déterminer l'effort de contact « archet/caténaire ».

\section{Validation de la méthodologie}

Cette méthodologie a été validée par un dispositif expérimental mis en ouvre en laboratoire. Elle est par la suite comparée à celle actuellement utilisée et dont l'archet est considéré indéformable.

Dans ce qui suit, la nouvelle méthodologie n'utilise que le premier mode élastique de l'archet.

\subsection{Caractéristiques intrinsèques de l'archet}

Cette section précise les résultats de l'analyse modale préalablement effectuée en «libre-libre» et ce, pour le type d'archet considéré pour la validation de la méthodologie (archet bi-bande type Duplex).

Cette analyse modale met en évidence que le premier mode de déformation dans un plan vertical est la torsion de l'archet $(68,7 \mathrm{~Hz})$. Ce mode a une réponse dynamique significative seulement si l'effort de contact sur la bande $\mathrm{N}^{\circ} 1$ est différencié de celui sur la bande $\mathrm{N}^{\circ} 2$. Ce n'est pas le but recherché dans notre cas (cf. paragraphe 3.2), c'est pourquoi le mode qui sera considéré pour la suite de l'analyse correspond à la première flexion de l'archet mesurée à $79 \mathrm{~Hz}$.

\subsection{Instrumentation}

Le dispositif est constitué d'un vérin hydraulique dynamique, dont la tête est équipée d'éléments de contact de caténaires (photo 1). Ces éléments sont fixés sur des cellules d'efforts permettant une mesure directe de la force injectée. La réponse dynamique de l'archet est mesurée en trois points à l'aide d'accéléromètres dont l'analyse est limitée à leur composante verticale.

- Instrumentation de la nouvelle méthode (Fig. 3) :

- deux accéléromètres placés au niveau des boîtes à ressort $\left(\ddot{z}_{1}\right.$ et $\left.\ddot{z}_{2}\right)$;

- deux accéléromètres placés au centre de l'archet (un sur chaque bande de frottement - $\ddot{z}_{3}$ et $\ddot{z}_{3 \text { bis }}$ );

- mesure d'effort dans les boîtes à ressort $\left(\mathrm{F}_{1}\right.$ et $\left.\mathrm{F}_{2}\right)$.

$N B$ : L'effort de contact est déterminé en faisant l'hypothèse que l'archet peut se déformer.

- Instrumentation de la méthode « archet indéformable $»$ (Fig. 3) :

- deux accéléromètres placés au niveau des boîtes à ressort $\left(\ddot{z}_{1}\right.$ et $\left.\ddot{z}_{2}\right)$;

- mesure d'effort dans les boîtes à ressort $\left(\mathrm{F}_{1}\right.$ et $\left.\mathrm{F}_{2}\right)$.

$N B$ : L'effort de contact est déterminé en faisant l'hypothèse que l'archet est indéformable. 


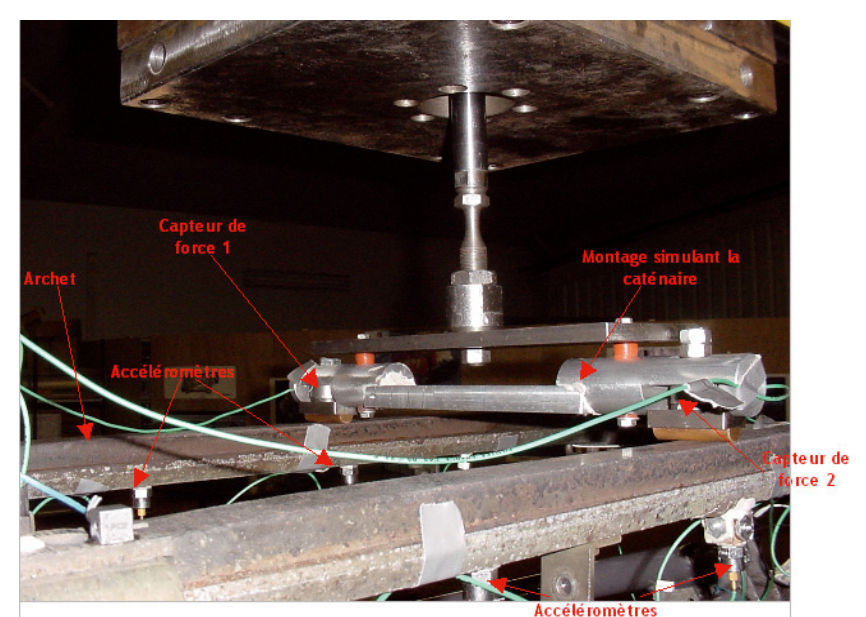

Photo. 1. Dispositif expérimental.

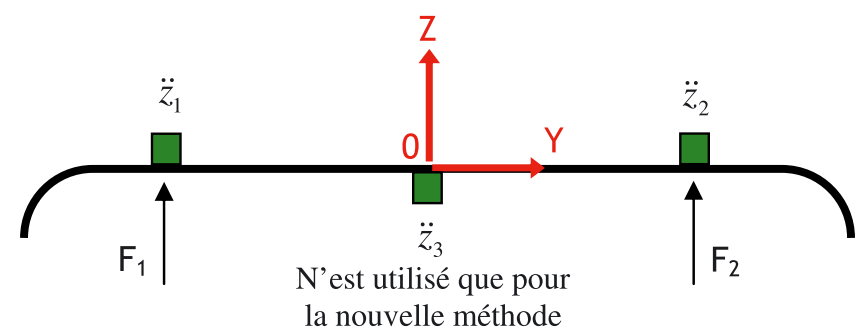

Fig. 3. Instrumentation utilisée pour la nouvelle méthode.

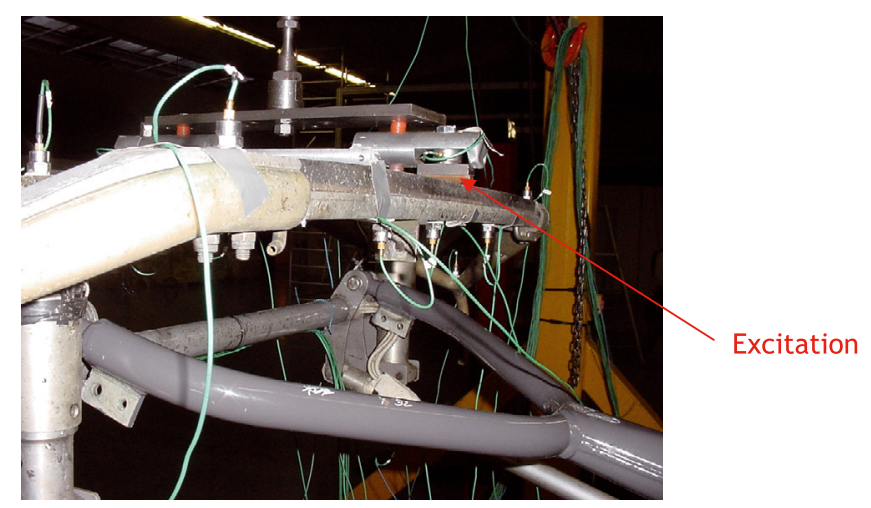

Photo. 2. Excitation au centre de l'archet.

\subsection{Excitations}

L'ensemble « archet + pantographe » est excité grâce au vérin hydraulique avec un signal de type « burst random » compris entre 0 et $200 \mathrm{~Hz}$. L'excitation varie de 6,13 N RMS autour d'un effort statique de $70 \mathrm{~N}$. L'archet est excité au centre des bandes de frottement (photo 2).

\subsection{Résultats pour un effort centré}

Les figures 4 et 5 comparent les efforts de contact reconstitués dans le domaine temporel et fréquentiel à partir des deux méthodologies.

Commentaires : L'analyse fréquentielle montre que de 0 à $45 \mathrm{~Hz}$, l'ancienne et la nouvelle méthode sont

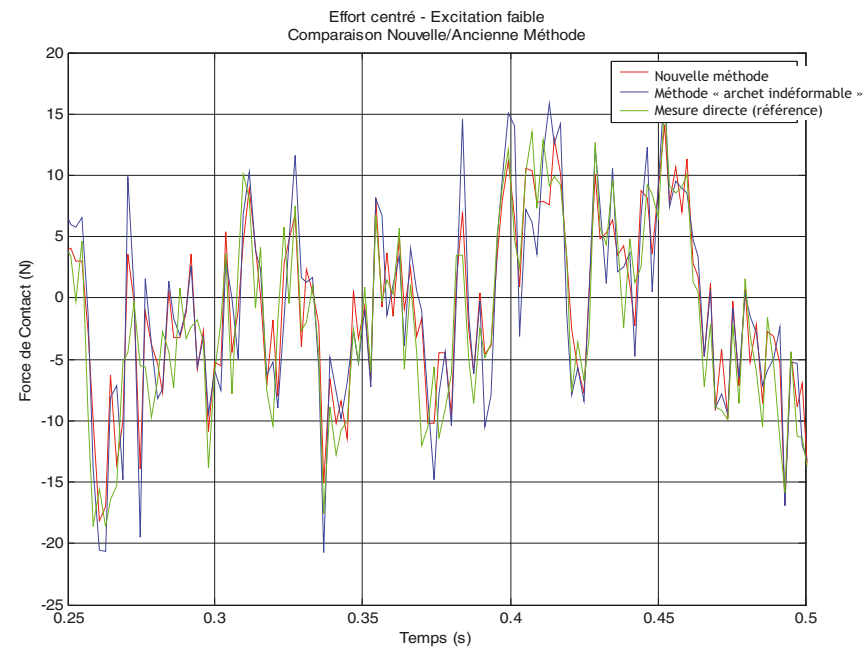

Fig. 4. Temporelle / effort centré / excitation faible.

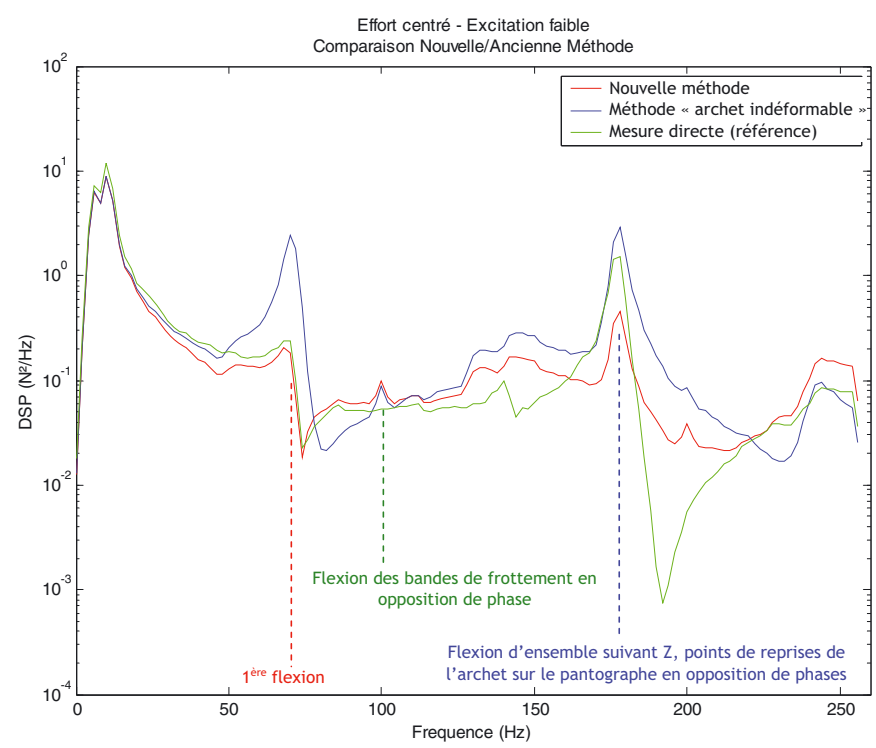

Fig. 5. DSP / effort centré / excitation faible.

équivalentes puisque l'archet est indéformable à ces fréquences.

Entre 50 et $120 \mathrm{~Hz}$, l'ancienne méthode diverge à cause de la présence du premier mode de flexion de l'archet. Cette divergence est totalement absente pour la nouvelle méthodologie.

Au-delà de $120 \mathrm{~Hz}$, les deux méthodes donnent un effort de contact erroné car les modes de fréquences supérieures ne sont pas pris en compte.

Conclusions : Les résultats obtenus, à partir du premier mode élastique de l'archet, montre un gain important sur la bande passante de la mesure, qui passe de $45 \mathrm{~Hz}$ à $120 \mathrm{~Hz}$ pour un archet dont la première flexion se situe aux environs de $75 \mathrm{~Hz}$. 


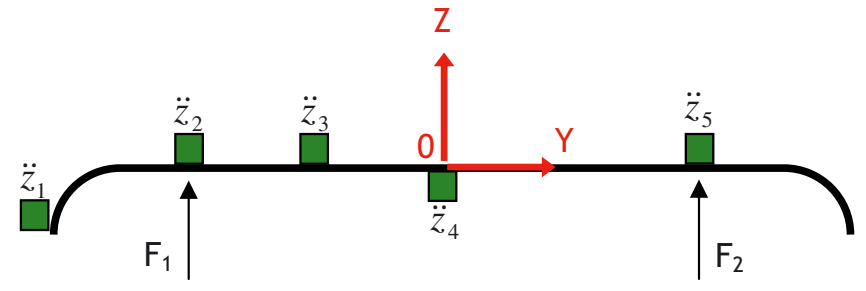

Fig. 6. Instrumentation.

Tableau 1. Configurations.

\begin{tabular}{cccccc}
\hline Configurations & $\ddot{z}_{1}$ & $\ddot{z}_{2}$ & $\ddot{z}_{3}$ et $\ddot{z}_{3 \mathrm{bis}}$ & $\ddot{z}_{4}$ et $\ddot{z}_{4 \mathrm{bis}}$ & $\ddot{z}_{5}$ \\
\hline Emplacement 1 & & $\checkmark$ & & $\checkmark$ & $\checkmark$ \\
\hline Emplacement 2 & $\checkmark$ & & & $\checkmark$ & $\checkmark$ \\
\hline Emplacement 3 & & $\checkmark$ & $\checkmark$ & $\checkmark$ \\
\hline Emplacement 4 & $\checkmark$ & $\checkmark$ & & $\checkmark$ \\
\hline
\end{tabular}

\subsection{Influence de l'emplacement des accéléromètres}

Afin de déterminer la pertinence des emplacements des accéléromètres, plusieurs configurations ont été testées pour déterminer l'effort de contact.

\subsubsection{Instrumentation}

L'instrumentation pour déterminer la pertinence des emplacements des accéléromètres se compose de :

- mesure d'effort dans les boîtes à ressort $\left(\mathrm{F}_{1}\right.$ et $\left.\mathrm{F}_{2}\right)$;

- deux accéléromètres placés au niveau des boîtes à ressort $\left(\ddot{z}_{2}\right.$ et $\left.\ddot{z}_{5}\right)$;

- deux accéléromètres placés au centre de l'archet $\left(\ddot{z}_{4}\right.$ et $\left.\ddot{z}_{4 \mathrm{bis}}\right)$;

- deux accéléromètres placés entre le centre de l'archet et une boîte à ressort $\left(\ddot{z}_{3}\right.$, et $\left.\ddot{z}_{3 \text { bis }}\right)$;

- un accéléromètre placé sur une corne $\left(\ddot{z}_{1}\right)$.

L'emplacement de ces différents capteurs est illustré en figure 6 .

Les différentes configurations sont obtenues en posttraitant un certain nombre de ces capteurs. Le tableau 1 synthétise les différents accéléromètres utilisés pour chaque configuration.

\subsubsection{Résultats}

La figure 7 montre que l'emplacement 2 donne la plus mauvaise corrélation entre 0 et $120 \mathrm{~Hz}$. Ce mauvais résultat est expliqué par le mode de torsion de l'archet qui n'a pas été pris en compte dans les équations. Les déplacements modaux au niveau des cornes sont très importants pour ce mode.

Le post-traitement des autres emplacements montre que les efforts de contact calculés sont sensiblement les mêmes jusqu'à la fréquence du premier mode de flexion.

Il en résulte que les accéléromètres doivent être placés sur les bandes de frottement ou sur les boîtes à ressort afin d'obtenir les meilleurs résultats.

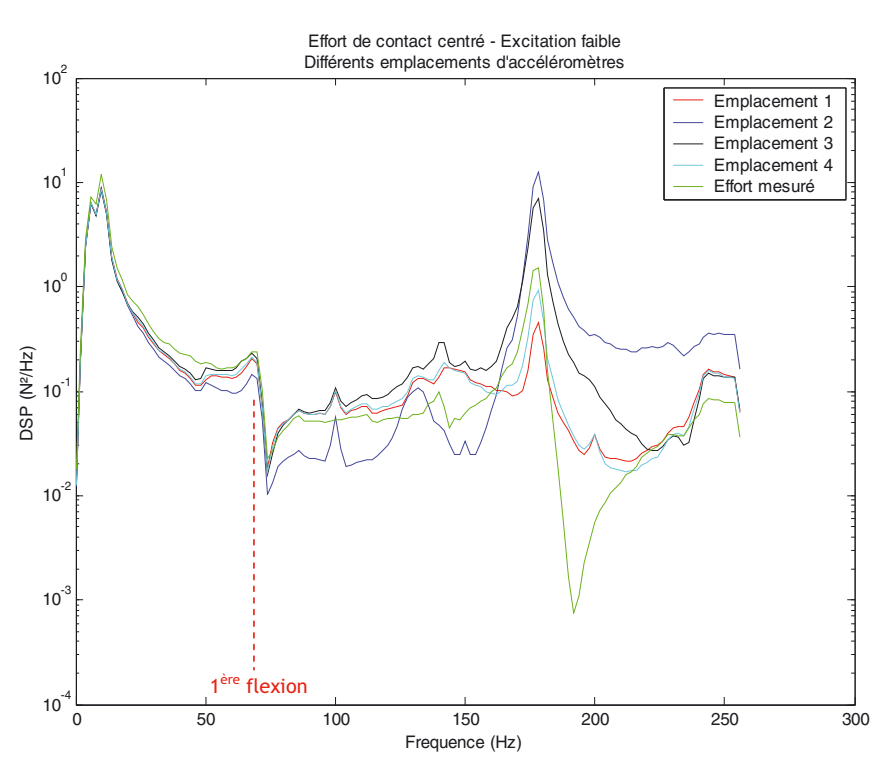

Fig. 7. DSP / effort centré / excitation faible.

Tableau 2. Configurations.

\begin{tabular}{cccccc}
\hline Configurations & $\ddot{z}_{1}$ & $\ddot{z}_{2}$ & $\ddot{z}_{3}$ et $\ddot{z}_{3 \text { bis }}$ & $\ddot{z}_{4}$ et $\ddot{z}_{4 \text { bis }}$ & $\ddot{z}_{5}$ \\
\hline Emplacement 1 & & $\checkmark$ & & $\checkmark$ & $\checkmark$ \\
\hline Surdétermination 1 & & $\checkmark$ & $\checkmark$ & $\checkmark$ & $\checkmark$ \\
\hline Surdétermination 2 & $\checkmark$ & $\checkmark$ & $\checkmark$ & $\checkmark$ & $\checkmark$ \\
\hline
\end{tabular}

\subsection{Surdétermination}

La surdétermination permet d'augmenter le nombre d'équations à résoudre tout en gardant le même nombre d'inconnues. Une optimisation du système est alors possible (choix des équations, méthode de régularisation, ....). Est-il nécessaire d'avoir recours à la surdétermination pour améliorer la nouvelle méthode? Deux configurations ont été envisagées pour répondre à cette question et sont comparées à la configuration de référence « emplacement $1 \%$.

\subsubsection{Instrumentation}

L'instrumentation est identique à l'étude sur les emplacements des accéléromètres (Fig. 6). Les différentes configurations sont obtenues en post-traitant un certain nombre de ces capteurs. Le tableau 2 synthétise les différents accéléromètres utilisés pour chaque configuration.

\subsubsection{Résultats}

La figure 8 montre que la surdétermination n'améliore pas la précision de la méthode dans la bande fréquentielle [0-120 Hz].

Quitte à utiliser plus d'accéléromètres, il nous semble préférable d'enrichir la base modale par des modes d'ordre supérieur. La précision de la nouvelle méthode sera accrue en haute fréquence. 


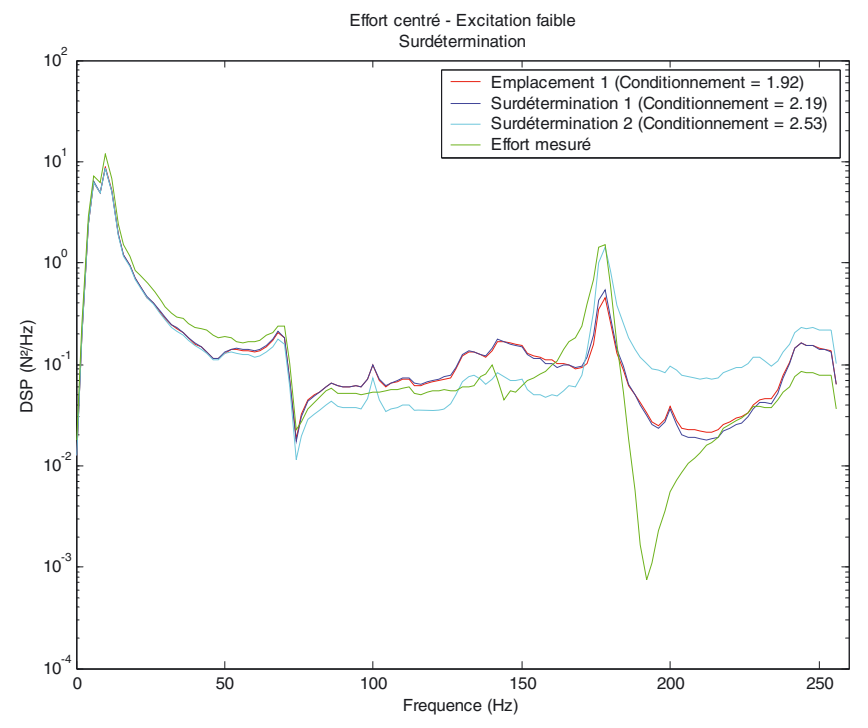

Fig. 8. DSP / effort centré / excitation faible.

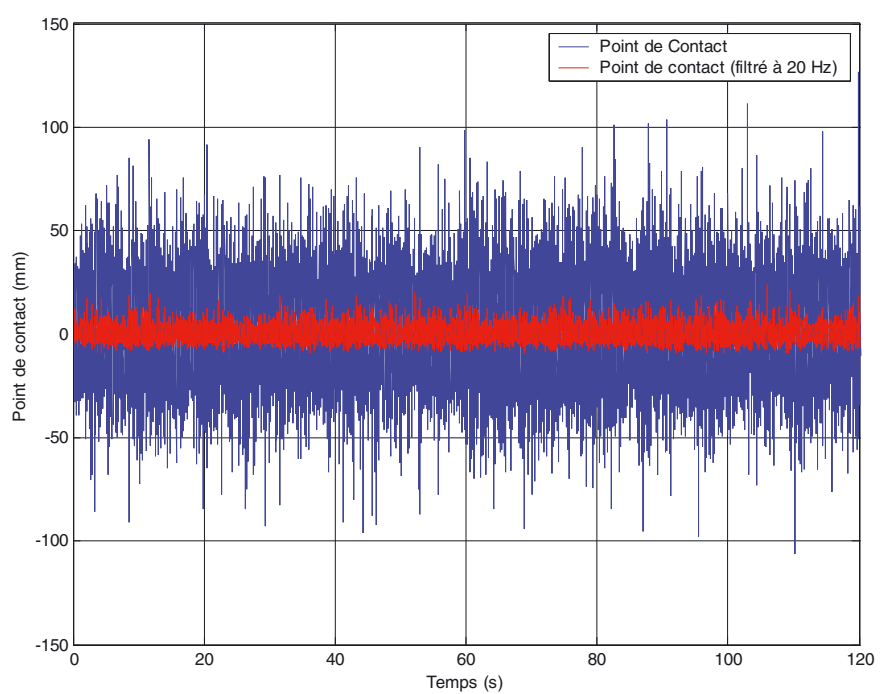

Fig. 9. Localisation du point de contact en fonction du temps - effort situé au centre de l'archet $(y=0 \mathrm{~m})$.

\subsection{Détermination du point de contact}

La détermination du point de contact s'effectue sans surdétermination. L'instrumentation de l'archet est la même que la configuration de référence « emplacement 1 ».

Les points de contact montrés dans les figures 9 et 10 ont été calculés à partir d'excitations faibles (sans décollement du contact) localisées respectivement au centre et sur un côté de l'archet.

Si le signal (courbes bleues) n'est pas filtré par un « passe-bas », la localisation du point de contact est estimée à $\pm 50 \mathrm{~mm}$; les bandes de frottement ont une longueur totale de $680 \mathrm{~mm}$ environ.

Ces erreurs sont importantes et sont principalement dues à une mauvaise approximation de l'effort de contact à haute fréquence; il est donc nécessaire de filtrer le signal par un filtre « passe-bas ». Une fréquence de coupure

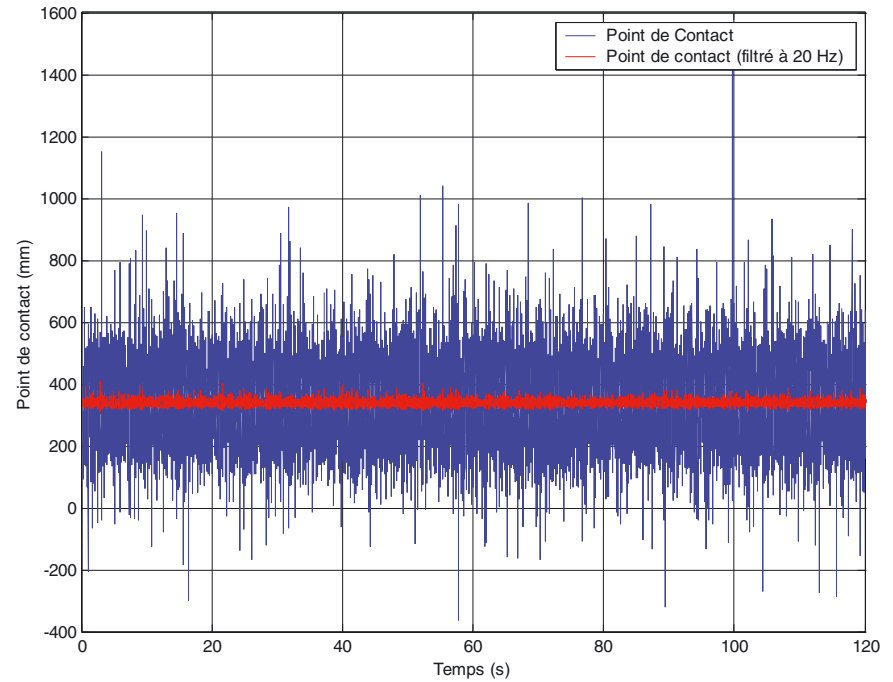

Fig. 10. Localisation du point de contact en fonction du temps - effort situé sur un côté de l'archet $(y=338 \mathrm{~mm})$.

à $20 \mathrm{~Hz}$ est choisie car la fréquence de déplacement du contact sur l'archet est inférieure à cette fréquence de coupure.

À partir de ce signal filtré (courbes rouges), la localisation du point de contact est notablement améliorée et est estimée à $\pm 15 \mathrm{~mm}$ environ.

\section{Conclusions}

La méthodologie proposée est une méthode inverse s'appuyant sur le principe de superposition modale. Pour pouvoir l'appliquer, une analyse modale expérimentale de l'archet doit être préalablement effectuée afin d'extraire les principales caractéristiques dynamiques de l'archet, à savoir :

- ses déformées modales en libre-libre;

- ses caractéristiques massiques.

L'effort de contact est déduit de mesures en ligne et à partir des caractéristiques modales de l'archet. L'instrumentation envisagée est la suivante :

- trois accéléromètres placés sur l'archet;

- un capteur d'efforts au niveau de chacune des deux «boîtes à ressort ».

Cette nouvelle méthodologie apporte de bonnes corrélations dans le domaine fréquentiel au-delà du premier mode de flexion. La méthode diverge à proximité des modes d'ordre supérieur, mais peut aisément être améliorée en prenant en compte ces modes. Il faudra, dans ce cas, ajouter des accéléromètres supplémentaires sur l'archet.

Cette nouvelle approche permettra de détecter d'éventuels défauts de caténaire (pendules défectueux...) grâce à la signature vibratoire de l'effort de contact mesuré. 


\section{Références}

[1] M. Ikeda, S. Nagasaka, A.U. Takayuki, Precise contact force measuring method for overhead catenary system, Railway Technical Research Institute, 2003

[2] R.R.J. Craig, M.C.C. Bampton, Coupling of Substructures for Dynamic Analysis, AIAA Journal 6 (1968) 1313-1319

[3] S. Teppe, C. Clerc, A. Bobillot, F.G. Rauter, Modélisation du Comportement Dynamique d'un Pantographe, XVe Colloque Vibrations Chocs et Bruit, 2006

[4] M. Aboshi, M. Manabe, Analyses of contact force fluctuation between centenary and pantograph, Quarterly Report of RTRI, Vol. 41, No 4, pp. 182-187, 2000
[5] C. Pezerat, Méthode d'identification des efforts appliqués sur une structure vibrante par résolution et régularisation du problème inverse, Thèse de l'INSA de Lyon, Réf ISAL010, 1996

[6] P. Bouvet, G. Lemaire, D. Thompson, P. Gauch, Quantification of dynamic forces applied by engine fuel injector by inverse methods, Société Française d'Acoustique, 2005

[7] N. Fremion, S. Teppe, O. Verin, C. Clerc, Minimisation des Bruits et Vibrations générés par les Machines Tournantes, XVIe Colloque Vibrations Chocs et Bruit, 2008 